Article

\title{
Influence of Different Structure and Specification Parameters on the Propagation Characteristics of Optical Signals Generated by GIL Partial Discharge
}

\author{
Xiaoxin Chen ${ }^{1}$, Chen $\mathrm{Li}^{1}$, Liangjin Chen ${ }^{2}$, Hui Wang ${ }^{3, *}$, Yiming Zang ${ }^{3}$ and Weijia Yao ${ }^{3}$ \\ 1 State Grid Zhejiang Electric Power Research Institute, Hangzhou 310014, China; shawcine@live.com (X.C.); \\ zljlxf1@outlook.com (C.L.) \\ 2 State Grid Zhejiang Electric Power Co. Ltd., Hangzhou 310007, China; liwang939@outlook.com \\ 3 Department of Electrical Engineering, Shanghai Jiao Tong University, Shanghai 200240, China; \\ zangyiming@sjtu.edu.cn (Y.Z.); vic.yao@sjtu.edu.cn (W.Y.) \\ * Correspondence: wanghui8203@sjtu.edu.cn; Tel.: +86-159-6660-8618
}

Received: 12 May 2020; Accepted: 9 June 2020; Published: 23 June 2020

\begin{abstract}
Partial discharge (PD) leads to the generation of electrical, acoustic, optical, and thermal signals. The propagation characteristics of optical signals in gas insulated metal-enclosed transmission lines (GIL) are the basis of optical detection research. This paper simulates the propagation of PD optical signals in GIL through modeling GIL with different structures and specification parameters. By analyzing the optical parameters on the probe surface and the detection points when the PD source position is different, the influence of the difference in specifications caused by the voltage level on the propagation of the GIL PD optical signal is studied. The results show that the GIL cavity structure will affect the faculae distribution and the relative irradiance (RI) of the detection surface; the PD source position has a huge impact on the faculae distribution on the detection surface, but has little influence on the RI; as the voltage rises, the faculae distribution on the detection surface becomes more obvious, and the mean of RI decreases. The above results have the reference value for the manufacture of GIL equipment and the research of PD optical detection. When the specular reflection coefficient of surface material is smaller and the diffuse reflection coefficient is larger, the outline of the light spot is clearer, the proportion of brighter parts is larger, and the maximum value of the RI is larger.
\end{abstract}

Keywords: partial discharge; optical signal; GIL; specification parameter; propagation characteristics; relative irradiance

\section{Introduction}

As the economy and industry develop rapidly, long-range and high-capacity power transmission technology has received increasing emphasis [1]. Traditional power transmission methods are susceptible to environmental factors such as wind, snow, and lightning. Gas insulated metal-enclosed transmission lines (GIL) are increasingly used because of the high capacity, low loss, and strong anti-interference ability [2-6]. According to previous experiments, when there are early insulation faults in the GIL, partial discharge (PD) often occurs with it $[7,8]$. Consequently, online monitoring for $\mathrm{PD}$ is of great significance in guaranteeing GIL safety during operation.

Optical detection is adequate for the GIL PD monitoring due to the totally sealed structure. This new PD detection technology for power equipment possesses strong anti-interference ability [9]; thus, it has received worldwide attention. Some scholars have executed preliminary researches including the corona spectrum in air (or insulating oil) [9-11], photoelectric effect theories [12,13], conception of fluorescent fiber sensors [14-16], and establishment of optical sensing systems [17-20]. Researchers $[21,22]$ have carried out simulation and research on the propagation characteristics of PD 
optical signals in GIS, which is of great significance for the field application and result analysis of optical detection. However, there have been no studies on the propagation characteristics of partial discharge light signals in GIL. This paper draws on the research ideas above and applies the research methods and simulation ideas to the partial discharge detection of GIL.

At the same time, this paper draws on the research ideas of Ultra High Frequency (UHF) signals, performs simulation modeling on GILs with different structures and voltage levels, and observes the optical parameters of the optical signals on the detection surface and detection points generated by partial discharge. The effects of the structure, the location of the partial discharge source, the voltage level and surface material on the PD optical signal propagation in GIL were analyzed. The results are the basis of future research on PD optical detection, and can be also referred during the manufacture of GIL.

\section{Simulation Settings}

Firstly, establish the simulation models of single-core and triple-core GIL cavities. The model is $1.25 \mathrm{~m}$ long, and has a shell with radius of $226 \mathrm{~mm}$, a wall with a thickness of $10 \mathrm{~mm}$, and an conductor with a radius of $45 \mathrm{~mm}$. The positions of the detection surfaces, detection points, and PD source are shown in Figure 1.

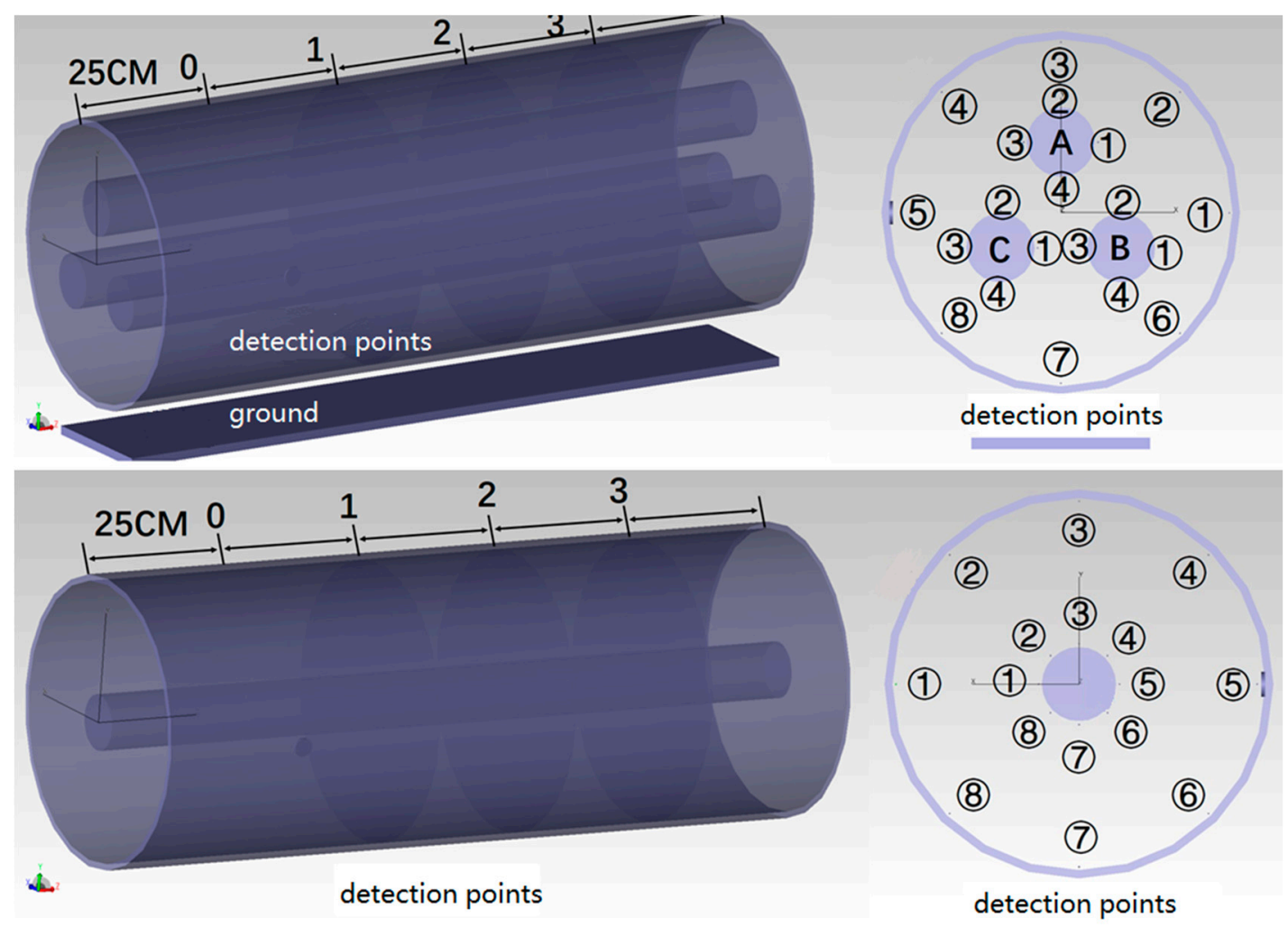

Figure 1. Simulation models of single-core and triple-cores gas insulated metal-enclosed transmission lines (GIL) cavities.

As shown in the left part of Figure 1, a detection point is fixed $25 \mathrm{~cm}$ from the left end on the tube wall. The circular intersecting surface at this position is regarded as the initial detection surface marked with 0 in Figure 1 . Then, others are set every $25 \mathrm{~cm}$, with marks of 1, 2, and 3 . The detection surfaces are circular rings with a radius of $15 \mathrm{~mm}$. The models of detection points and surfaces are fully transmissive, which means no absorption or refraction of the light can occur. In the triple-core GIL cavity, eight sources are installed around the inside of the shell and four sources around the outside of every internal conductor for every detection surface, as shown in the upper right corner in 
Figure 1. There are four surfaces in total; thus, 80 source positions are set up. In the single-core GIL cavity, eight sources are evenly installed along the inside of shell and eight sources along the outside of internal conductor for every detection surface, as shown in the lower right corner in Figure 1. There are four surfaces in total; thus, 64 source positions are set up.

The design standards and structural dimensions of GIL vary with the voltage levels [23,24]. Based on the proposition in paper [24,25], six sets of parameters for triple-core GIL under different voltage levels are listed in Table 1.

Table 1. Parameters of triple-cores GIL models under different voltage levels.

\begin{tabular}{ccccccc}
\hline Voltage/kV & $\mathbf{1 2 6}$ & $\mathbf{2 5 2}$ & $\mathbf{3 6 3}$ & $\mathbf{5 5 0}$ & $\mathbf{8 0 0}$ & $\mathbf{1 1 0 0}$ \\
\hline internal radius of the shell/mm & $\mathbf{2 2 6}$ & 292 & 363 & 496 & 637 & 743 \\
diameter of internal conductor/mm & 90 & 102 & 127 & 178 & 178 & 203 \\
insulation distance/mm & 69 & 95 & 118 & 159 & 236 & 270 \\
\hline
\end{tabular}

The radiance of the light signal of the partial discharge indicates the amount of radiation of the sensor, which equals to the received luminous flux per unit area, which can be used to indicate the strength of the optical signal received by the sensor:

$$
E_{e}=\frac{d \Phi_{v}}{d S}
$$

where $S$ represents the light receiving area and $\Phi_{v}$ represents the received luminous flux. The optical signal generated by partial discharge is a pulse signal. Considering the light-emitting shape of the pin-plate discharge model, when the discharge voltage is not large, only the discharge occurs near the tip of the needle. At this time, the light source is nearly spherical, and the partial discharge only occurs in a very small space near the insulation defect. Therefore, we can simulate it as a point light source during simulation [22]. In this paper, we used optical simulation software tracepro to perform simulation experiments. A spherical point light source is selected as the discharge source. The discharge source emits light in a direction perpendicular to the surface and in a quantity of 100 watts of luminous flux. It should be noted that the optical power parameters of the pointolite simulation model and the actual PD source is different. As a result, this article acquired relative irradiance (RI), which represents a relative value according to the real irradiance. RI cannot explain the real irradiance, but its change tendency is the reflection of the real irradiance variation.

In Sections 2-4, the surface material of the body is selected from polished and oxidized medium smooth aluminum, with an absorptivity of $30 \%$, a specular factor of $20 \%$, and a diffuse reflectivity of $50 \%$. The diffuse reflection model adopts a bidirectional reflection distribution function (BRDF). In Section 5, there are two different surface material properties.

\section{Influence of Structure on Propagation Characteristics of GIL PD Signals}

\subsection{Study on the Faculae of the Detection Surface}

First, study the faculae on different detection surfaces while the PD occurs. The faculae image refers to the irradiance analysis chart of the detection surface, which can reflect the light radiation intensity distribution. Due to the different positions of the light source, different shapes of light faculae are formed after multiple reflections in the cavity. It can be seen that the strength of the light signal at different positions reflects the light propagation characteristics, not the light faculae formed by lens elements. This paper only analyzed the faculae on the detection surfaces when the PD sources are located at position marked with 0 . The situations of other locations are too similar to need be described in this paper. The RI increases as the faculae color becomes bright (yellowish red).

Figure 2 is the image of the light faculae on detection surfaces marked with 1,2, and 3 of single-core GIL cavity. The partial discharge source is located at the (1)-8) position of the shell surface and the (1)-8) 
position of internal conductor surface. Each line represents the light faculae for a partial discharge source in different positions of the same detection surface. The number in parentheses means the largest value in the RI of this line.

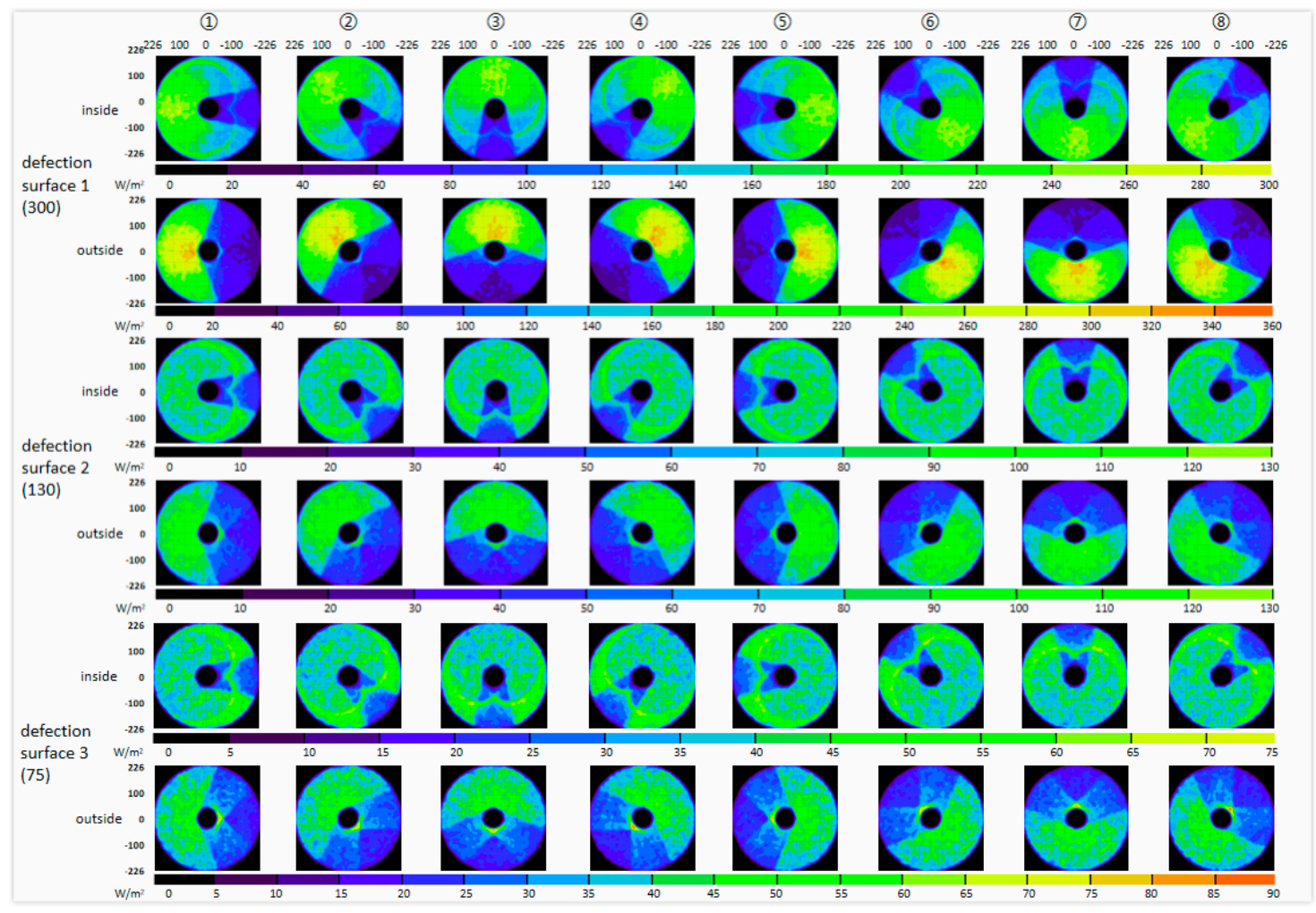

Figure 2. The faculae on the detection surfaces of the single-core GIL.

It can be seen that the farther the detection surface is, the darker the faculae are, the more obscure the contour is, and the more even the distribution is. This phenomenon can be explained as follows: for farther detection surfaces, the effect of the direct light is diminished, while the effect of reflected light is enhanced; thus, the light received by the surface is weakened, but the faculae are evened out. The value of RI decreases sharply with distance, too.

The faculae are symmetrical, and this property always exists no matter where the discharge source is located. The reason is that the single-core GIL cavity has a structure of centrosymmetry. Thus, the symmetry of faculae remains unchanged both under the single discharge source and multiple sources with a symmetrical distribution. At the same time, the internal conductor obstructs the propagation of the light emitted by some partial discharge sources, resulting in a small RI value in this area, resulting in dark faculae on the detection surface.

However, the PD source position has a certain effect on the light faculae. On the condition that the sources are located around the inside of shell, the fan-shaped area exceeds that on the condition of source locating on the outside of the internal conductor. The bigger the brighter area is, the more homogeneous the color is, and the smaller the maximum RI is. In addition, if the PD sources are located around the inside of the shell, a linear facula could be observed on the detection surface. However, this phenomenon does not occur if the PD sources are located around the outside of the internal conductor. The reason is speculated to be multiple reflections. The light in the former situation needs a further radial travel than that in the latter situation, thereby leading to a line-shaped light facula on the detection surface.

Then, for the simulation experiment of the triple-core GIL model, the partial discharge sources were respectively on the inside of shell and the outside of three internal conductors. The obtained 
analysis shows that it is the same as the single-core GIL. As the distance from the detection surface augments, the faculae get more even, the color gets darker, and RI suffers a precipitous drop. When they are close to the PD location, the faculae color gets brighter and RI gets a raise. However, the symmetry of the light faculae is different from the central symmetry of the single-core GIL, but possesses an axisymmetry. Only if the partial discharge sources are located on the symmetry axis, the light faculae on the detection surface have the mutual symmetry. Compared to single-core GIL, three internal conductors in triple-core GIL obstruct more direct light emitted by the partial discharge source. As a result, the dark area of the light faculae is greater.

When the partial discharge source is on the inside of the shell, the brighter area is significantly larger than that when the partial discharge source is on the outside of internal conductor, but the maximum RI is lower, which resembles the situation of single-core GIL. At the same time, the shielding effect of the internal conductor also changes if the PD source location changes.

\subsection{Analysis of Relative Irradiance on Detection Surface}

Figure 3 exhibits the mean RI value measured on the detection surface during the variation of PD source position in the single-core and triple-core GIL cavities. This indicates that the RI has a precipitous drop with the rising distance of the detection surface. This result is in accordance with the analysis of the faculae.

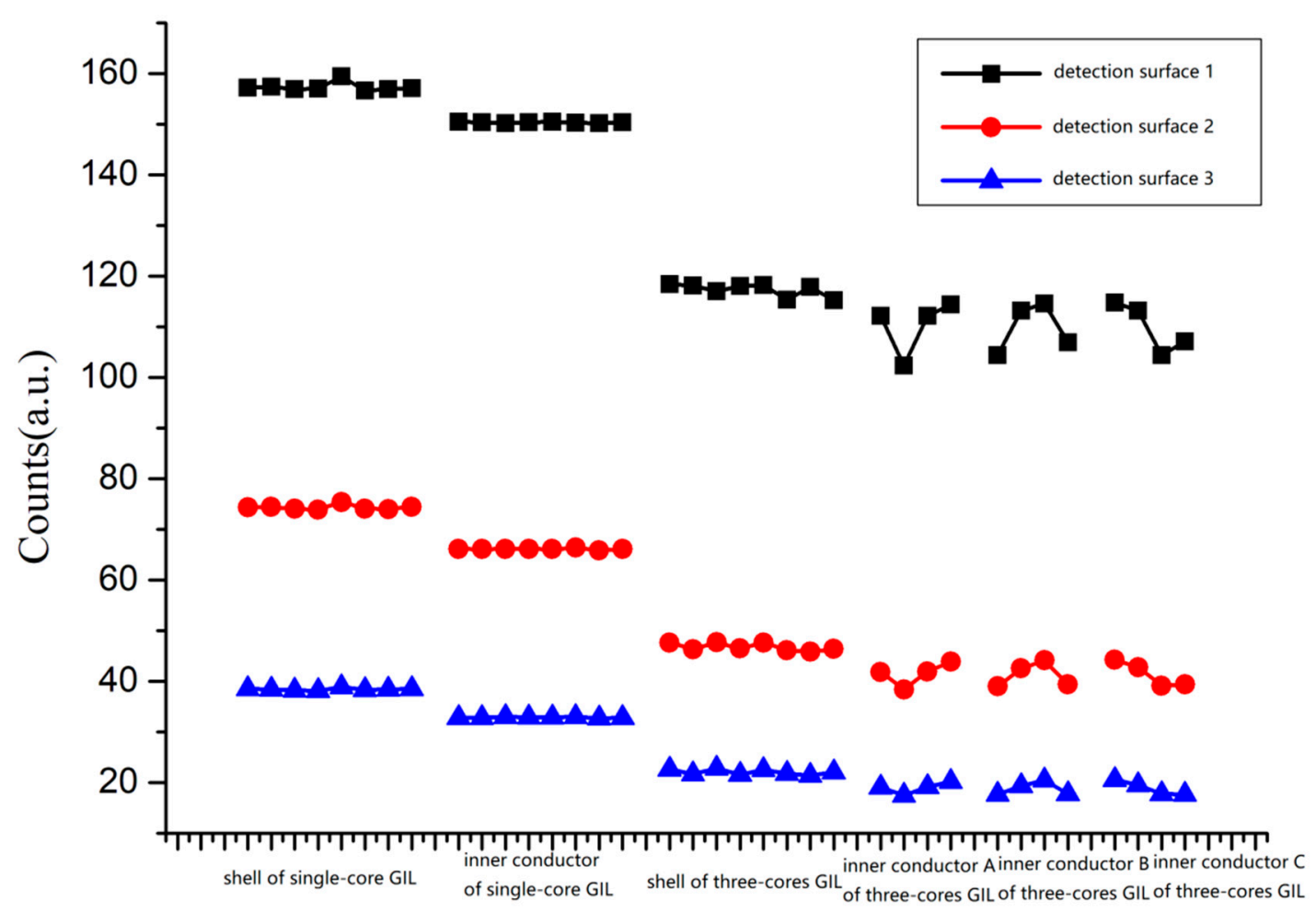

Figure 3. Average relative irradiance on the detection surfaces.

Moreover, RI keeps the same level if the PD source position does not change. RI reaches the maximum when the PD sources are located around the inside of the single-core shell, followed by the outside of the single-core internal conductor, followed by the inside of the triple-cores shell. The minimum value is reached on the outside of the triple-cores internal conductor. For the first three situations, the RI line fluctuates slightly, indicating that the sources at distinct angles at this time has a slight impact on the mean RI. However, in the last situation, the line fluctuates greatly, indicating that the sources at distinct angles have a greater impact at this time. 


\subsection{Analysis of Relative Irradiance at Fixed Detection Points}

Figure 4 exhibits the RI value measured at fixed detection point marked with 0 , while the PD source locations vary around the inside of the single-core GIL shell (No. 0, 1, 2, and 3). Because when the partial discharge source is at position (5) of section 0 , the RI value of the detection point (66,590 a.u. (astronomical unit)) is far greater than other situations. In fact, it amounts to discharging the probe, which directly leads to the breakdown of the probe; thus, the above figure excludes this situation.

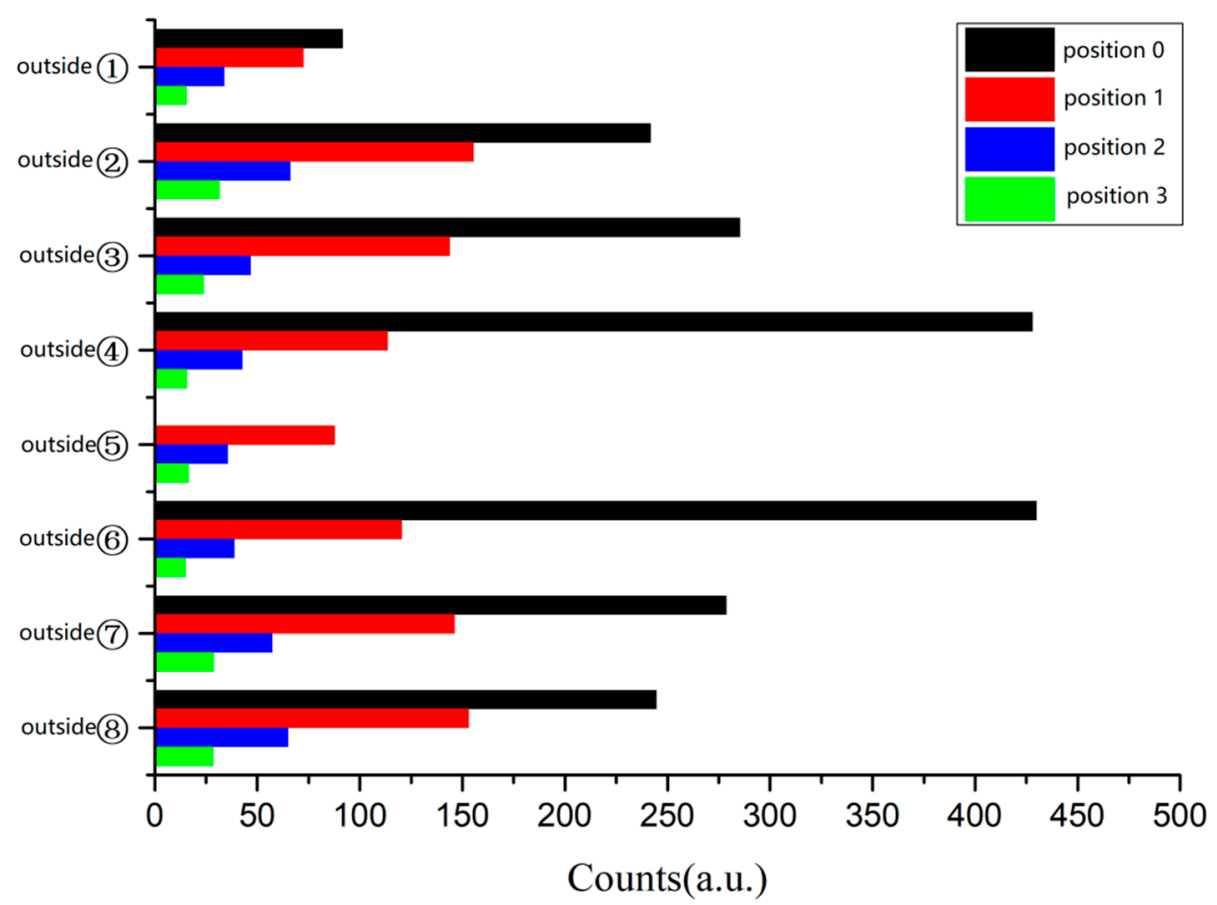

Figure 4. Relative irradiance values at fixed detection point.

The above figure indicates that if the sources are located at the same angle but at distinct horizontal positions, the height of the bar graph decreases and the RI value decreases with the rising horizontal distance. The further the detection point is, the more RI slightly decreases. At the same time, because of the symmetrical structure of the single-core GIL, the distribution of RI values has certain symmetry if only the angles change while the horizontal location of PD sources does not change. However, the horizontal location will influence the characteristics.

If the source locations are distinct on the internal surface of the single-core GIL (on the 0, 1, 2, and 3 cross sections), the RI decreases as the distance increases, and also exhibits a certain symmetry. This phenomenon resembles the situation of inside of the single-core GIL shell. Differently, the extent to which the PD sources at the same point decrease with distance increases is less different from each other.

If the PD sources are placed around the inside of the triple-core GIL shell, the RI images were studied in the same way and compared with the RI images of the single-core GIL. In the triple-core GIL, the characteristic that the RI value decreases with the increase of the PD source distance still exists, but there exist no strong signs of regularity in the range of decrease. At the same time, the RI value obtained by the partial discharge source around the inside of the shell in the triple-core GIL also has some symmetry.

If the PD sources are placed around the outside of three internal conductor in triple-core GIL, the RI measured at detection points basically keep the same, with slightly different details.

Figure 5 summarizes the RI values of the detection points when the PD source is at each point around the shell inside in the single-core GIL and triple-core GIL. The figure indicates that though 
the single-core GIL and the triple-core GIL possess distinct structure of internal conductor, they share some resemblance with the RI generated by the sources around the inside of the shell.

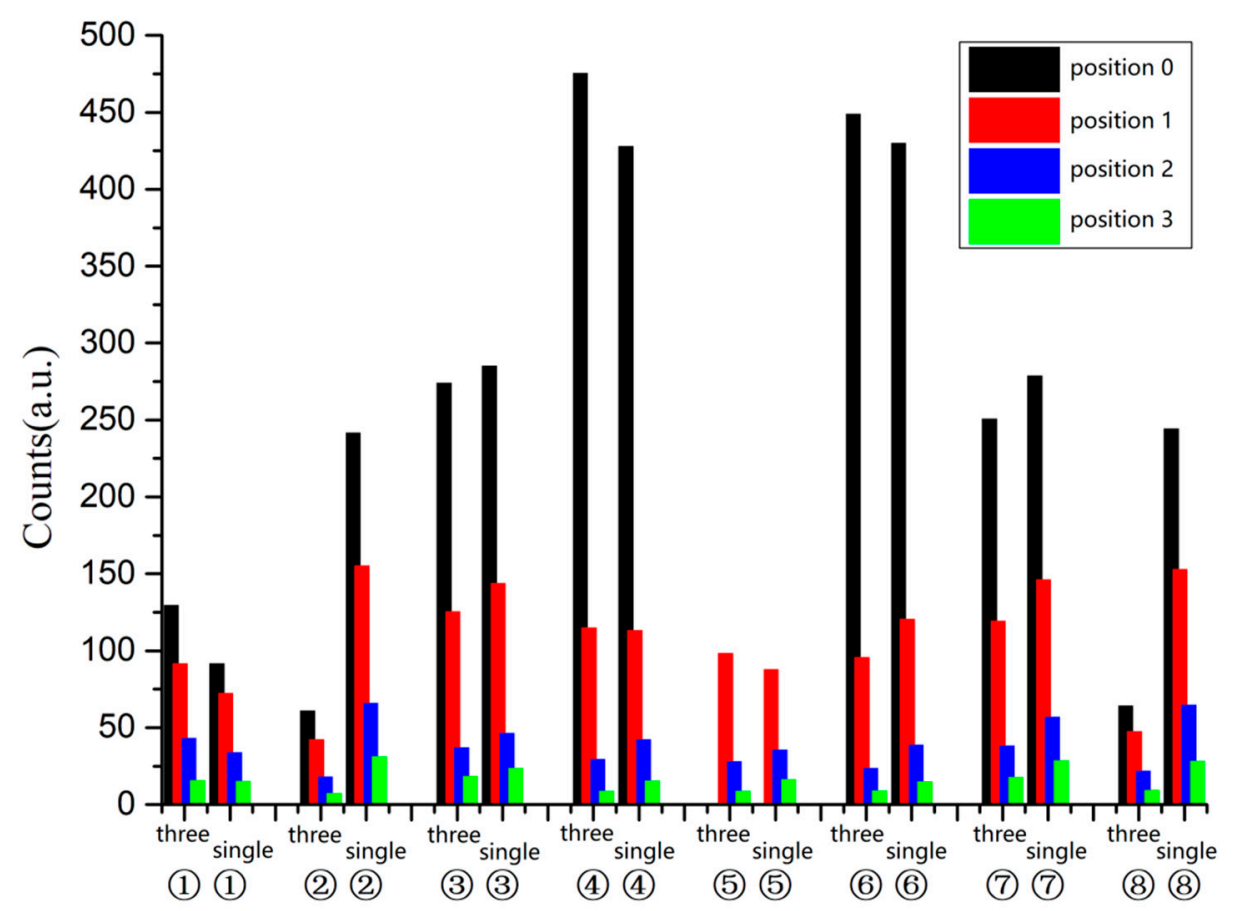

Figure 5. Comparison of relative irradiance values.

\section{Influence of the PD Sources' Position on Propagation Characteristics of GIL PD Signals}

\subsection{Study on the Faculae of the Detection Surface}

Figure 6 is the image of the light faculae on the detection surface 1 and 3 when the PD source is around the outside of three internal conductors in the GIL363 cavity. Because the triple-core GIL cavity possesses axisymmetry, the faculae arose from the PD sources in the symmetrical position on the detection surface is also symmetrical. Thus, part of the faculae are not shown in the above figure.

Figure 6 indicates that as the distance of the detection surface increases, the outline of the faculae gets gradually blurred, and the distribution becomes more even. The reason is that the direct light reaching to the detection surface weakens. At the same time, the reflected light gets strengthened to homogenize the light spot. The value of irradiance decreases sharply with distance.

The color of the faculae near the PD source position is yellowish green, that is, the RI value in this area is large. Moreover, the three internal conductors blocked the direct light emitted from the PD source, leading to a dark facula on the detection surface. Consequently, the RI value in this area is very small.

Comparing it with the images when the partial discharge source positions are around the inside of the shell, it can be seen that the bright spot areas are significantly smaller when the partial discharge sources are located around the outside of internal conductor. This is because the internal conductor obstructs the direct light from the partial discharge source, and the difference of source positions affects this shielding degree. 


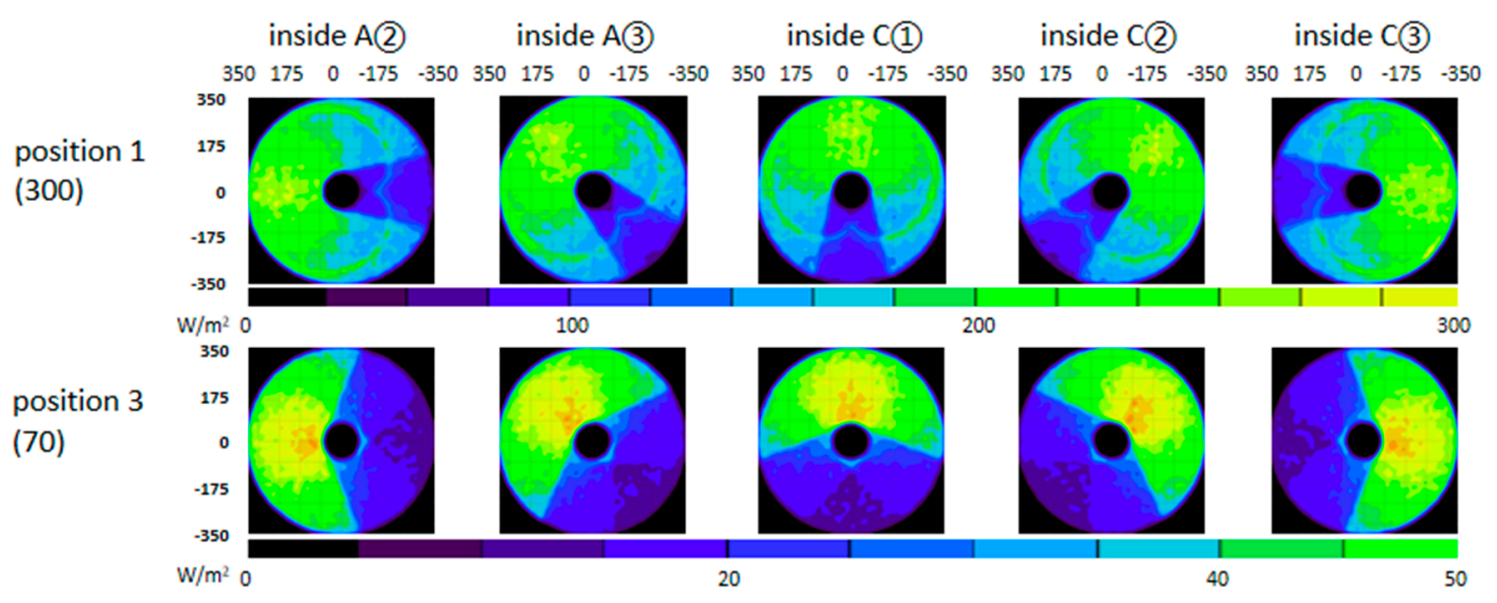

Figure 6. The faculae on the detection surfaces of GIL363.

\subsection{Analysis of Mean Relative Irradiance on Detection Surface}

It was concluded during the simulation that when the PD sources were installed at distinct points around the inside of the shell with the same circular intersecting surface, or at different positions around the outside of the internal conductors with the same circular intersecting surface, the average RI value of the detection surface was relatively close. Take the GIL126 cavity model for instance, and put the PD sources at distinct points on surface marked with 0 ; the mean of the RI values is then obtained, as shown in Table 2.

The result indicates that although the location of the partial discharge sources change, the average RI values of the detection surface are very close, and the coefficients of variation are less than $10 \%$.

Table 2. Average relative irradiance values on GIL126 detection surfaces.

\begin{tabular}{|c|c|c|c|c|c|c|c|c|}
\hline & & Position 1 & Position 2 & Position 3 & Position 4 & Position 5 & Position 6 & Position 7 \\
\hline GIL & Average & 117.27 & 46.82 & 22.26 & 12.17 & 7.39 & 4.88 & 3.41 \\
\hline $\begin{array}{c}126 \\
\text { Outside }\end{array}$ & $\begin{array}{l}\text { Standard } \\
\text { Deviation }\end{array}$ & 1.30 & 0.75 & 0.53 & 0.36 & 0.27 & 0.20 & 0.16 \\
\hline \multirow{4}{*}{$\begin{array}{c}\text { GIL } \\
126 \\
\text { Inside }\end{array}$} & $\begin{array}{l}\text { Coefficient of } \\
\text { Variation }\end{array}$ & $1.11 \%$ & $1.60 \%$ & $2.39 \%$ & $2.98 \%$ & $3.58 \%$ & $4.00 \%$ & $4.58 \%$ \\
\hline & Average & 109.97 & 41.41 & 19.05 & 10.19 & 6.10 & 3.99 & 2.77 \\
\hline & $\begin{array}{l}\text { Standard } \\
\text { Deviation }\end{array}$ & 4.58 & 2.24 & 1.18 & 0.72 & 0.45 & 0.33 & 0.24 \\
\hline & $\begin{array}{l}\text { Coefficient of } \\
\text { Variation }\end{array}$ & $4.17 \%$ & $5.40 \%$ & $6.18 \%$ & $7.07 \%$ & $7.36 \%$ & $8.25 \%$ & $8.54 \%$ \\
\hline
\end{tabular}

\subsection{Analysis of Relative Irradiance at Fixed Detection Points}

Figure 7 shows the change of the RI value at a fixed detection point if the PD sources are located at distinct points around the inside of shell in a single cavity (taking GIL363 as an example). Among them, when the partial discharge source is at position (5) of section 0 , the RI value $(95,926$ a.u.) is much larger than other cases; thus, it is not shown in the figure above. 


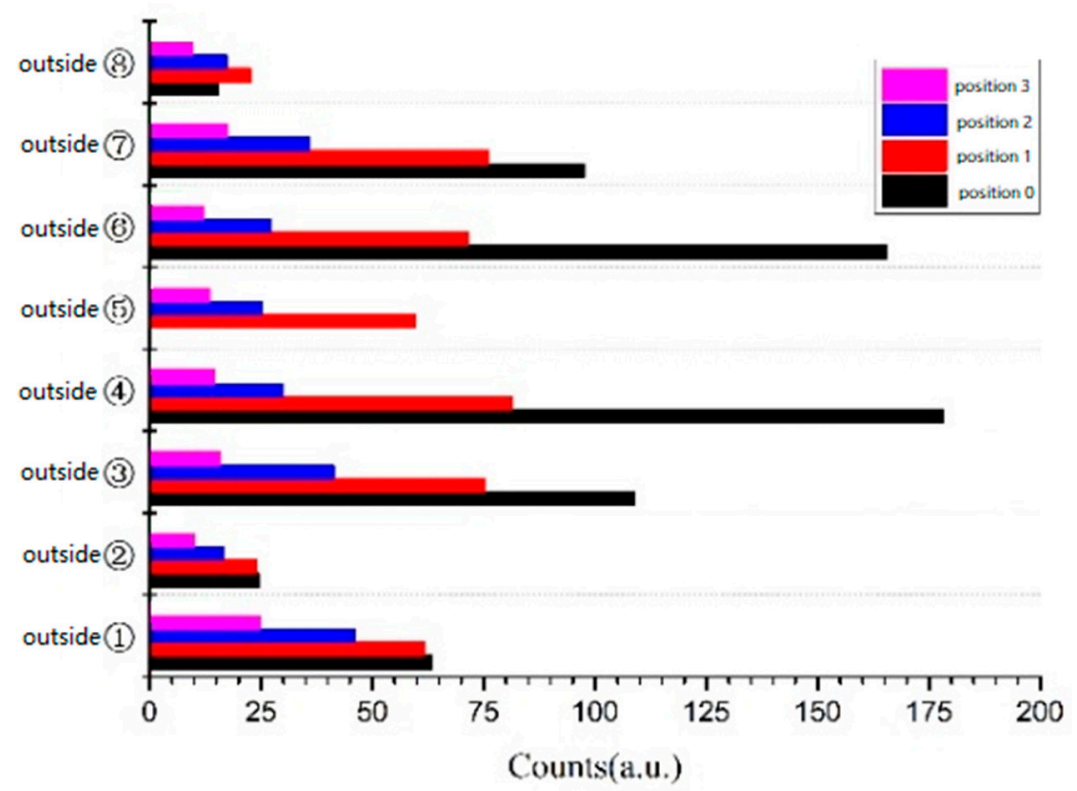

Figure 7. The relative irradiance at detection point of GIL363.

It can be seen that the RI value decreases with the distance of the PD source, but the degree of decrease does not reflect the obvious regularity.

If the PD sources are located at distinct points around the outside of internal conductor in the GIL363 cavity, the characteristics of RI value measured at a fixed detection point generally stay the same, but the details are slightly different: the line contours of the irradiance at distinct points share some similarity, but the points where the maximum RI value is obtained at each position are different. The common characteristics of the above-mentioned partial discharge sources are that horizontally, the fixed detection point is relatively close to the source; thereby, the light does not need to pass through many obstructions.

\section{Influence of GIL's Specifications on Propagation Characteristics of GIL PD Signals}

\subsection{Study on the Faculae of the Detection Surface}

Figure 8 is the faculae on detection surface No. 1 in the GIL cavity of different specifications when the partial discharge sources are located around the inside of the shell. It can be seen that in a low-voltage level GIL, such as GIL126, the color of the darker area of the faculae is dark blue; the maximum value of the RI spot is relatively large. In the high-voltage level GIL, the color of the darker area of the faculae is black, which means that the value of this area is far smaller than that of the above-mentioned area. The maximum of RI under the high-voltage GIL is smaller than that of the low-voltage level GIL. That is, under the condition of the same partial discharge source, as the voltage level increases, the maximum value of the RI on the detection surface gradually decreases, the contrast between the bright part and the dark part of the faculae is more distinct. This is mainly because the higher the voltage is, the larger the GIL cavity radius is, and the larger the internal conductor radius is. As a result, the longer the light propagation path from the PD source is, the more contrasting the faculae distribution appears on the detection surface, which also affects the maximum RI value.

If the PD sources are located around the outside of the internal conductor, similar conclusions can be reached after analysis, which are not repeated here. 


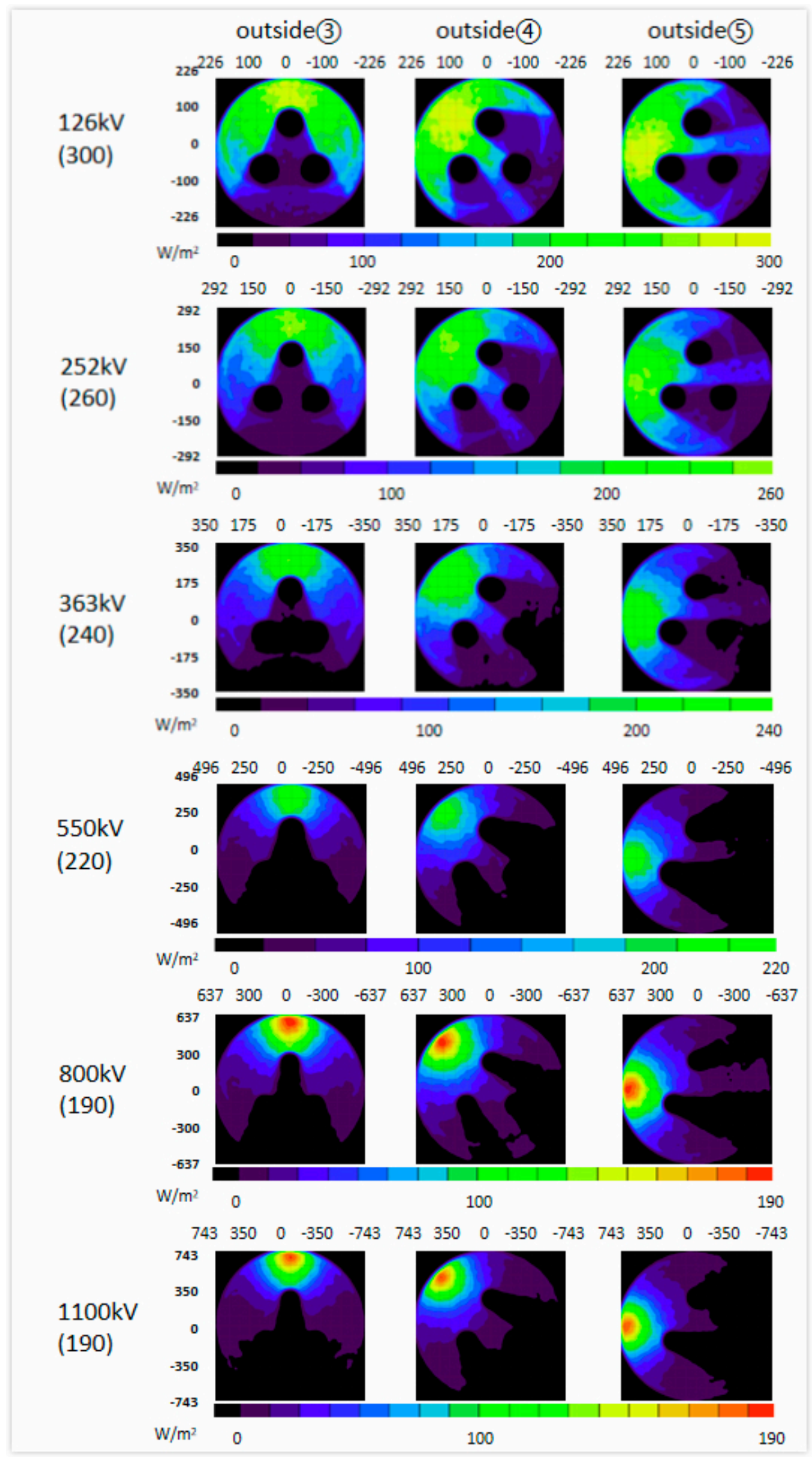

Figure 8. The faculae on detection surface No. 1 of different GILs. 


\subsection{Analysis of Mean Relative Irradiance on Detection Surface}

Figure 9 indicates that as the detection surface is farther away from the partial discharge source, the average RI value of the detection surface becomes smaller. The difference between the cavity models gradually decreases as the detection surface becomes farther. The above rules can be attributed to the effect of distance. The longer the path of optical signal propagation, the weaker the signal reaching the detection surface. The reason why the difference between the models is reduced is that the small magnitude of the values must be taken into account.

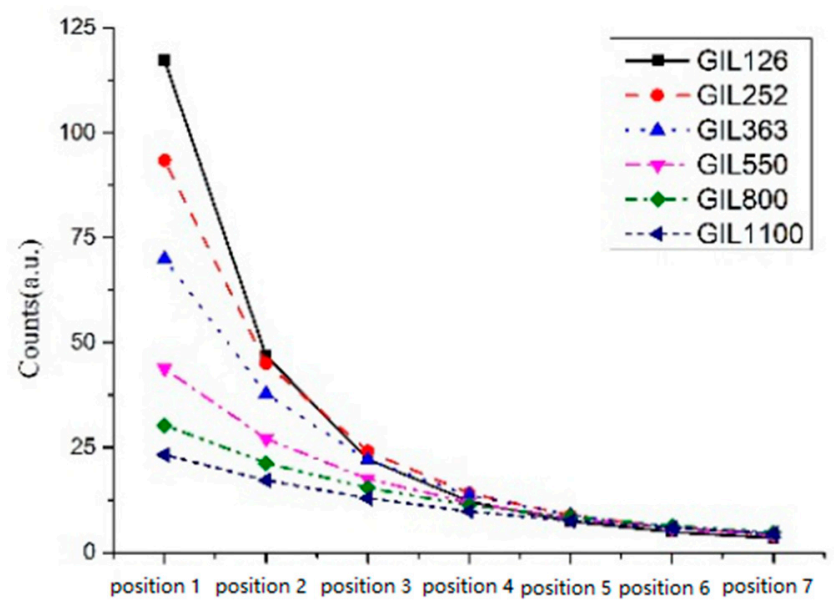

Figure 9. The mean of relative irradiance in different GILs.

The light reaching to the detection surface is mainly divided into two parts, one is the direct light, and the other is the reflected light reflected from the surface of the cavity to the surface of the detection surface. The RI value on the detection surface can be regarded as the joint effect of these two parts. The direct light part is greatly affected by the distance. With reference to the inverse-square law in geometric optics of PD sources on the surface of shell, the lines in Figure 9 can be approximated as an inverse proportional function. However, due to the existence of the reflection part, the RI value is approximately flat at a long distance, and this part is difficult to be represented by a simple formula. For related detector designs, the reflection part is the key.

Figure 10 shows the difference between the partial discharge sources around the inside of shell and the outside of the internal conductor. It indicates that when the distance from the detection surface becomes larger, the difference becomes closer, presenting a graph of convergence. At the same time, for models with higher voltage levels, the difference between the internal conductor and the case is smaller. Generally, the value on the condition of shell inside will exceed that of the outside of the internal conductor. However, in the GIL800 and GIL1100 models, the value of the surface of internal conductor at position 1 is larger than that of the shell. The reasons can be attributed to the following two points: one is the influence of the propagation distance, and the other is the effect of the smaller magnitude. 


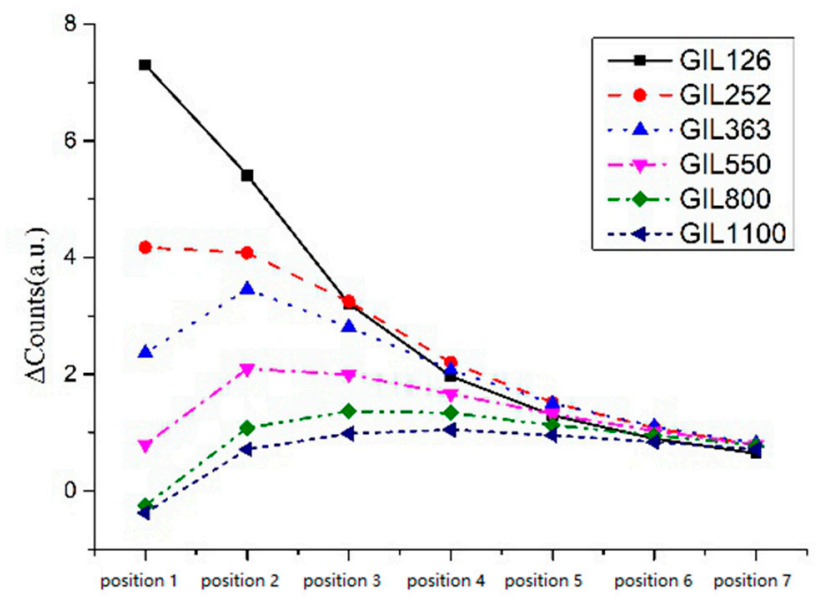

Figure 10. The difference between the average relative irradiance values.

\subsection{Study on Relative Irradiance at Fixed Detection Points}

When the PD sources are located at the No. 1 position in GIL models under different voltage levels, the RI values at fixed detection point are shown in Figure 11. We can see the similarities and differences between the Gil cavity models with different voltage levels. The similarities are that the contours of the lines formed by the RI values are generally similar. The difference is that the size relationship between some PD sources has changed, and is gradually reversed with the rise of the voltage level, even producing a gap. These phenomena are mainly caused by the structure and specifications. The same structure leads to similar changes, but the difference in specific specifications leads to changes in details.

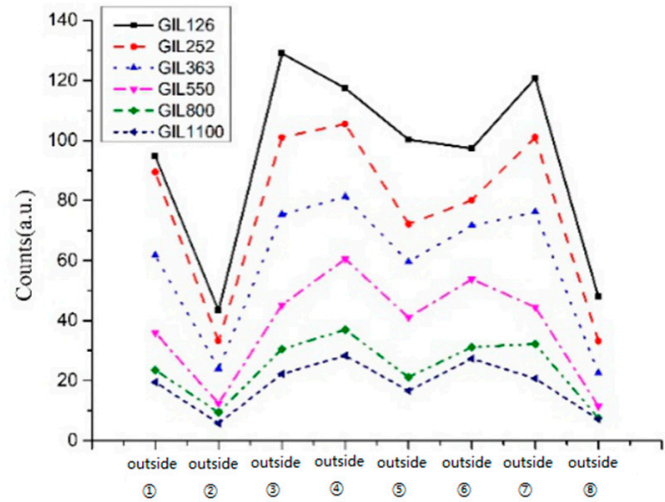

(a)

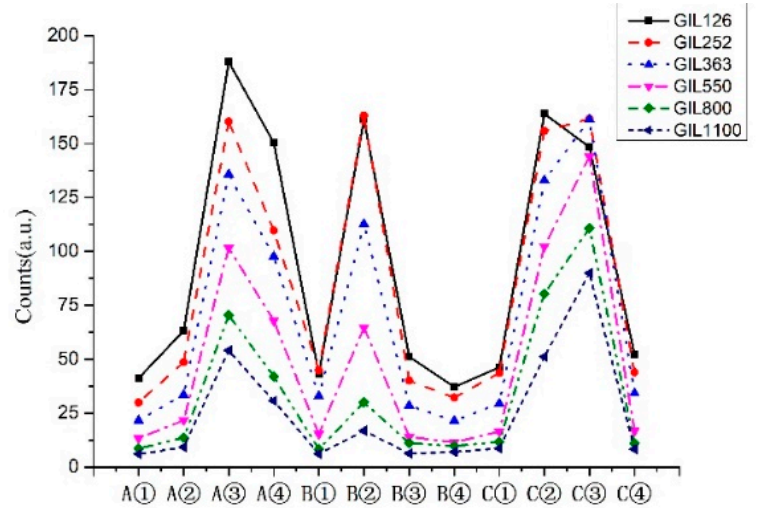

(b)

Figure 11. The relative irradiance at detection points: (a) detection points at the shell (b) detection points at the three internal conductors.

\section{Effects of the Surface Materials on Propagation Characteristics of GIL PD Signals}

\subsection{Study on the Faculae of the Detection Surface}

Figure 12 is the image of the light faculae on the detection surface 1 when the partial discharge sources are located at (1)-(8) position (a) around the inside of shell and (1)-(8) position (b) around the outside of internal conductor on the surface marked with 0 in GIL126 cavity. The left column represents the case where the absorption coefficient of the surface material is equal to $30 \%$, the specular factor is equal to $20 \%$, and the diffuse reflectivity is equal to $50 \%$; the right column represents the case where the absorption coefficient of the surface material is equal to $30 \%$, the specular factor is equal to $40 \%$, 
and the diffuse reflectivity is equal to $30 \%$. The diffuse reflection models are bidirectional reflection distribution functions (BRDFs).

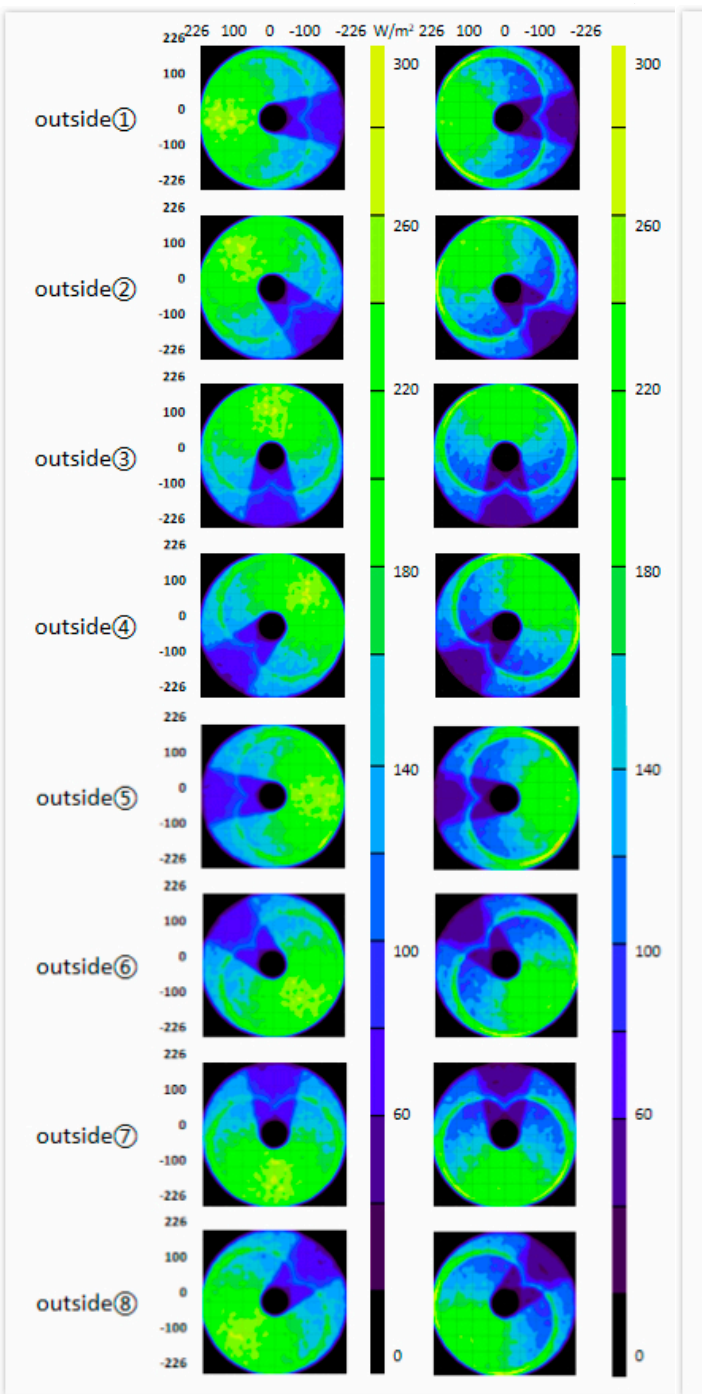

(a)

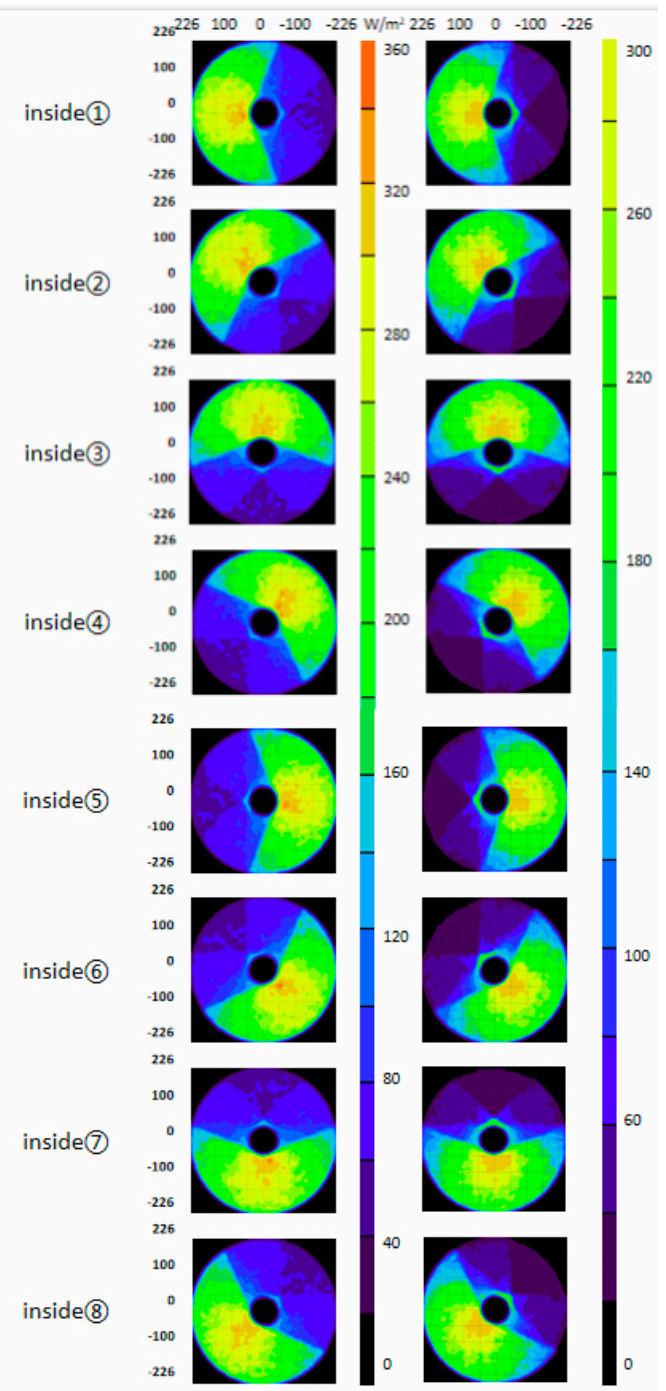

(b)

Figure 12. The faculae on the detection surface marked with 1, with different surface materials: (a) detection points at (1)-8) position of shell (b) detection points at (1)-8) position of internal conductor.

It can be seen that under the same absorption rate, the overall distribution of the faculae generated by the partial discharge source at the corresponding positions is similar, and the faculae color is close. However, when the specular factor is smaller and the diffuse reflectivity is larger, the outline of the light spot is clearer, the proportion of brighter parts is larger, and the maximum value of the RI is larger. When the specular factor is larger and the diffuse reflectivity is smaller, the light faculae distribution is more uniform, the darker portion accounts for a larger proportion, the line-shaped light faculae is more obvious, and there are also more obvious line-shaped light faculae on the detection surface farther away.

When analyzing the light faculae detected on detection surfaces 2 and 3, the comparison was similar to detection surface 1 , and will not be repeated here. 


\subsection{Study on Relative Irradiance on Detection Surface}

Figure 13 exhibits the RI values detected on detection surface 1 for the two surface materials when the source is located at distinct points in the single-core and triple-core GIL cavity. Figure 13 indicated that the trend of the curve is basically the same, but when the specular factor is equal to $20 \%$ and the diffuse reflectivity is equal to $50 \%$, the RI values detected on detection surface 1 are greater than the other case.

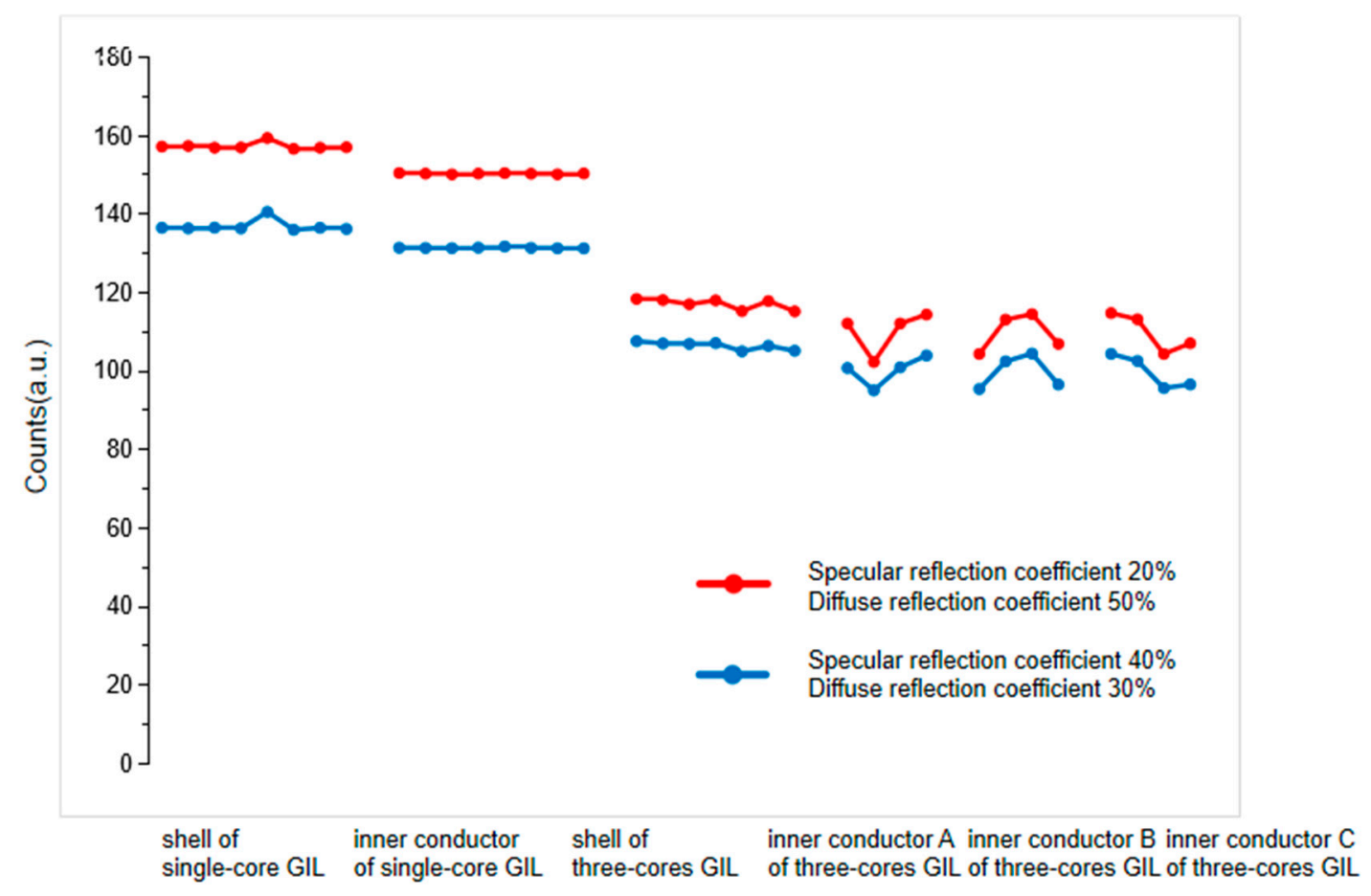

Figure 13. Relative irradiance values on detection surface 1.

Then, the RI values detected on detection surfaces 1-3 if the sources are located at distinct points in the single-core GIL under two surface materials are analyze. This situation is shown in Figure 14. Similarly, the curve trend is basically the same, but it need be noticed that the data of detection surface 3 has changed. When the specular factor is equal to $20 \%$ and the diffuse reflectivity is equal to $50 \%$, the RI values detected on detection surfaces 3 are less than in the other case.

It is known that the closer detection surface is greatly affected by direct light, and the farther detection surface is affected by reflected light. Therefore, it is shown that the specular reflection coefficient has no obvious effect on the optical signal on the closer detection surface, but has a greater effect on the detection surface that is further away.

Each segment of data in Figure 14 was averaged, and the data was normalized separately. It can be seen that the greater the specular reflection coefficient, the smaller the average relative irradiance values decrease with distance; the decrease is lower, that is, the attenuation is smaller. 


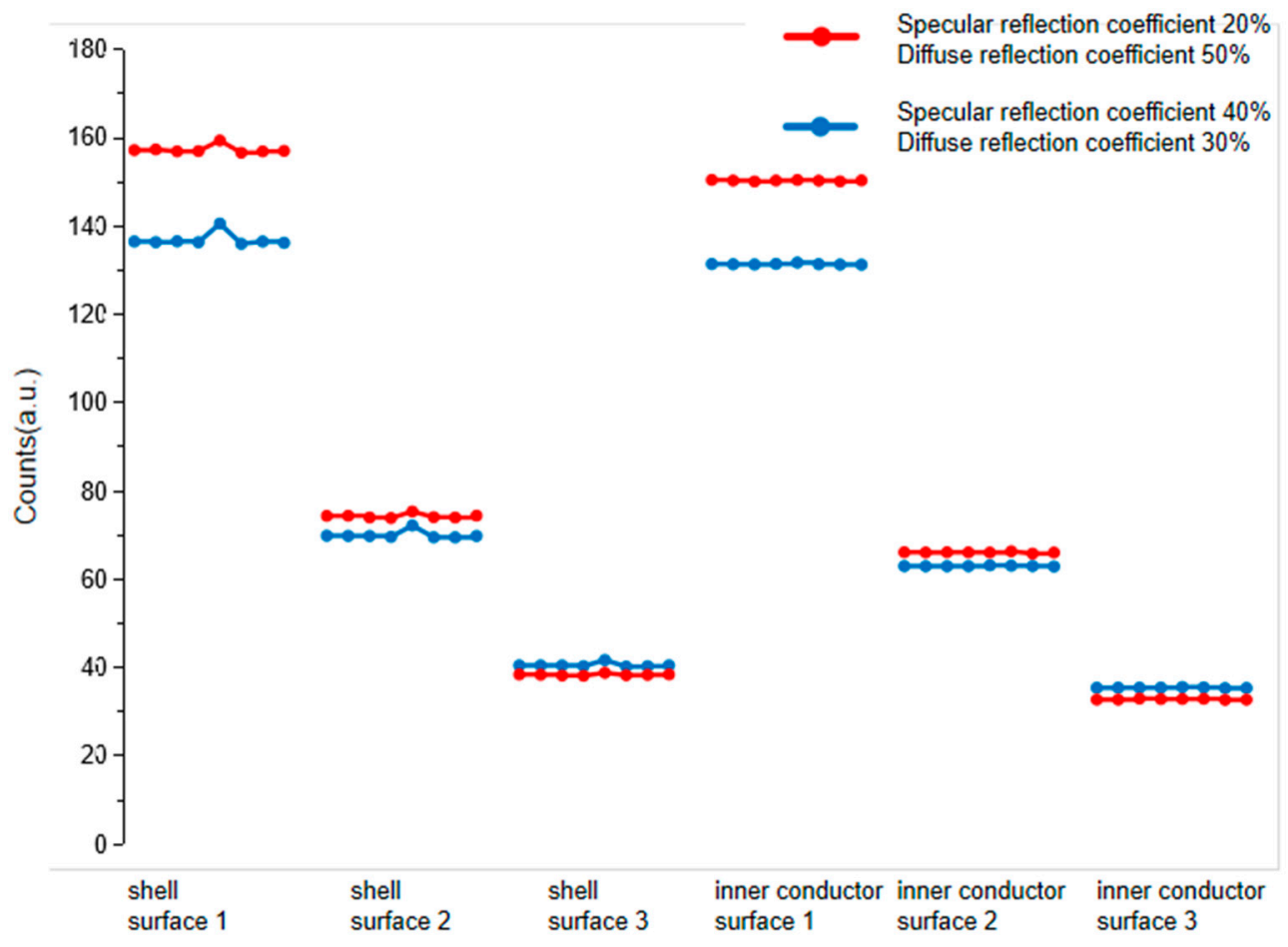

Figure 14. Relative irradiance values on 1-3 detection surface.

Figure 15 shows the results of averaging and normalizing each segment of data in Figure 14. It can be seen that the greater the specular reflection coefficient, the smaller the average relative irradiance values decrease with distance; the decrease is lower, that is, the attenuation is smaller.

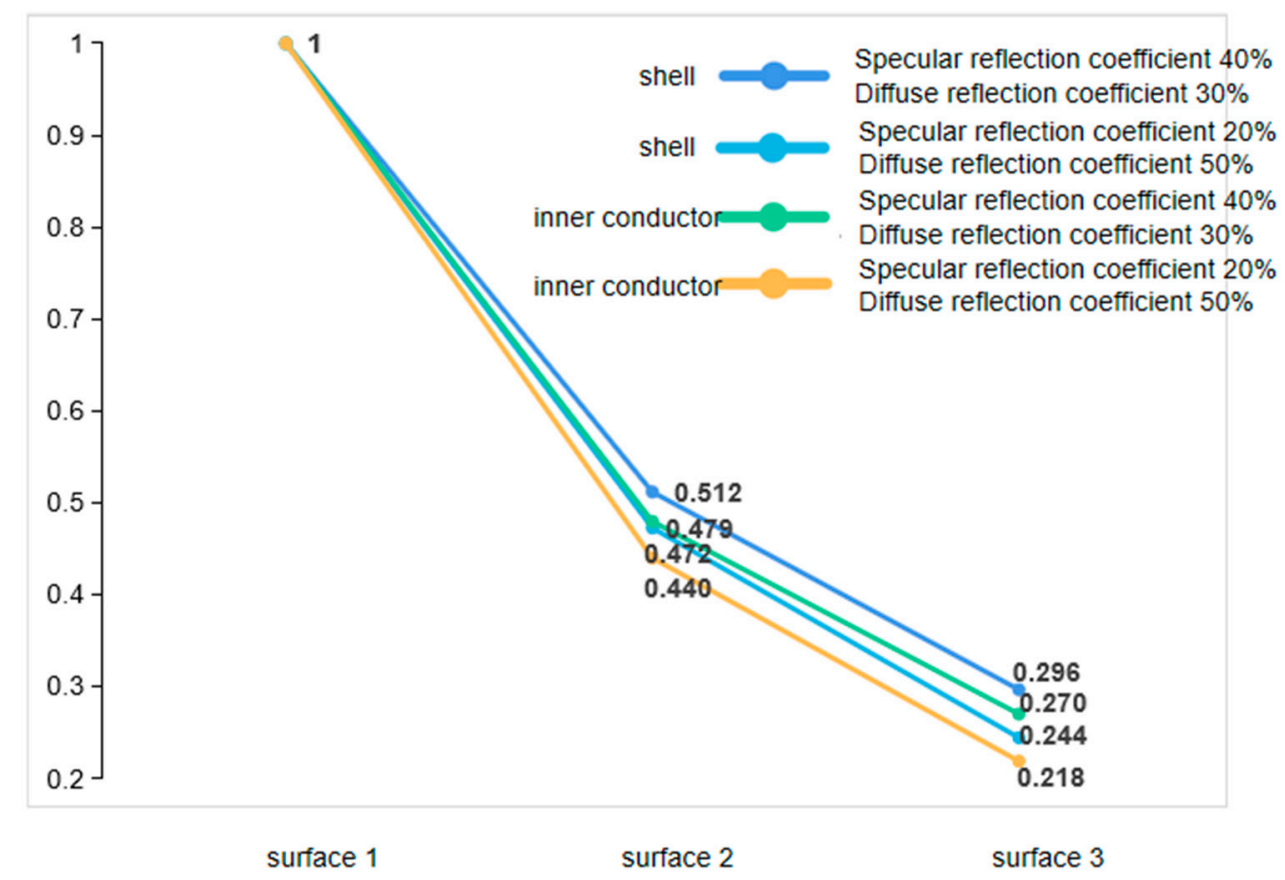

Figure 15. Attenuation of the relative irradiance values on detection surface 1-3. 


\subsection{Study on Relative Irradiance of Fixed Detection Points}

Table 3 shows the RI value and the difference rate detected at fixed detection point set in section 0 under two surface materials when the partial discharge source is located at the shell surface (1)-8) in section 0 .

Table 3. Relative irradiance values and difference rate at fixed detection point.

\begin{tabular}{ccccccccc}
\hline & & Point 1 & Point 2 & Point 3 & Point 4 & Point 5 & Point 6 & Point 7 \\
\hline GIL & 343 & 71.7730 & 213.670 & 272.140 & 419.120 & 65359.000 & 418.940 & 267.300 \\
126 & 325 & 91.597 & 241.730 & 285.200 & 427.860 & 66590.000 & 429.970 & 278.720 \\
Outside & difference rate & $28 \%$ & $13 \%$ & $5 \%$ & $2 \%$ & $2 \%$ & $3 \%$ & $4 \%$ \\
\hline
\end{tabular}

It can also be seen that when the specular factor is equal to $20 \%$ and the diffuse reflectivity is equal to $50 \%$, the RI value detected at fixed detection point is greater than other cases. The difference rate is between 2 and $28 \%$.

Although the RI value and the difference rate vary greatly, there is also symmetry. It can be seen that point 4 and point 6 , point 3 and point 7 , and point 2 and point 8 correspond to each other.

Similarly, when the sources are placed around the outside of the internal conductor at point (1)-8) in section 0 , the RI value and the difference rate also show the same rules, as shown in Table 4.

Table 4. Relative irradiance values and difference rate at fixed detection point.

\begin{tabular}{ccccccccc}
\hline & & Position 1 & Position 2 & Position 3 & Position 4 & Position 5 & Position 6 & Position 7 \\
\hline GIL & 343 & 48.799 & 88.212 & 323.900 & 627.940 & 803.500 & 693.360 & 338.490 \\
126 & 325 & 91.120 & 118.880 & 371.550 & 669.620 & 927.570 & 711.570 & 384.100 \\
Inside & difference rate & $87 \%$ & $35 \%$ & $15 \%$ & $7 \%$ & $15 \%$ & $3 \%$ & $13 \%$ \\
\hline
\end{tabular}

\section{Conclusions}

This article establishes both single-core and triple-core GIL models, and analyzes the effects of the GIL structure, partial discharge source position, voltage level, and surface material on the PD optical signal propagation. The conclusions are as follows:

1. Effect of GIL structure on optical signal: all the faculae of single-core GIL have center symmetry. In the triple-cores GIL, only the faculae produced by the symmetrically positioned PD source are symmetrical to each other. The average RI value on the detection surface decreases with the increase of the PD source distance.

2. The location of the PD source has a significant impact on the faculae on detection surface, but has little effect on the average RI of the detection surface. The RI value at a fixed detection point decreases as the distance from the PD source increases.

3. As the voltage level of GIL augments, the maximum value of RI on detection surface gradually decreases, and the contrast between the bright part of the faculae and the dark part increases. The difference between the average RI value on the detection surface of the internal conductor and the shell is reduced.

4. When the specular reflection coefficient of the surface material is smaller and the diffuse reflection coefficient is larger, the outline of the light spot is clearer, the proportion of brighter parts is larger, and the RI values detected on the detection surfaces and at a fixed detection point are larger.

Author Contributions: Conceptualization, X.C.; Data curation, X.C.; Formal analysis, X.C.; Funding acquisition, X.C.; Investigation, C.L.; Methodology, C.L.; Project administration, C.L.; Resources, C.L.; Software, L.C.; Supervision, L.C.; Validation, L.C.; Visualization, Y.Z.; Writing—original draft, H.W.; Writing—review and editing, W.Y. All authors have read and agreed to the published version of the manuscript.

Funding: This research received no external funding. 
Acknowledgments: Project supported by the Science and Technology Project of State Grid Zhejiang Electric Power Co., Ltd. (Optical GIS Partial Discharge Detection and Severity Assessment Technology 5211DS180035).

Conflicts of Interest: The authors declare no conflict of interest.

\section{References}

1. Qi, B.; Zhang, G.; Li, C.; Gao, C.; Zhang, B.; Chen, Z. Research Status and Prospect of Gas-insulated Metal Enclosed Transmission Line. High Volt. Eng. 2015, 41, 1466-1473.

2. Li, P.; Yan, X.; Wang, H.; Zhang, Q.; Jin, G.; Gao, Y.; Mu, S. Research and Application of UHVAC Gas-Insulated Transmission Line. Power Syst. Technol. 2017, 41, 3161-3167.

3. Zhang, C.; Han, D.; Li, K.; Zhang, G. SF6 Alternative Techniques and Their Applications and Prospective Developments in Gas Insulated Transmission Lines. High Volt. Eng. 2017, 43, 689-698.

4. Zhuo, R.; Tang, J.; Zhang, X.; Xiong, H.; Jiang, H.; Tang, J. Relationship Between UHF Signals and Discharge Quantity Under Various Pressures in Air Insulated Transmission Line. High Volt. Eng. 2014, 40, 1475-1480.

5. Xiao, D.; Yan, J. Application and Development of Gas Insulated Transmission Line (GIL). High Volt. Eng. 2017, 43, 699-707.

6. Wang, J.; Li, Q.; Li, B.; Liu, S.; Wang, Z. Motion and Discharge Behavior of the Free Conducting Wire-type Particle Within DC GIL. Proc. CSEE 2016, 36, 4793-4801.

7. Li, Q.; Liu, S.; Wang, J. Characteristics of Partial Discharges Caused by Free Metal Particle and Influencing Factors in DC Gas Insulated Line. High Volt. Eng. 2017, 43, 367-374.

8. Zhang, G.; Zhang, B.; Wang, Q.; Li, J. Experiment Study of Surface Charge Accumulation and Decay on a Cone-type Insulator in HVDC GIL. High Volt. Eng. 2015, 41, 1430-1436.

9. Fujii, K.; Yamada, M.; Tanaka, A.; Kurosawa, K. Emission spectrum of partial discharge light in SF6 gas. In Proceedings of the 1992 IEEE International Symposium on Electrical Insulation, Baltimore, MD, USA, 7-10 June 1992; pp. 332-335. [CrossRef]

10. Boczar, T.; Zmarzly, D. Optical spectra of surface discharges in oil. IEEE Trans. Dielectr. Electr. Insul. 2006, 13, 632-639. [CrossRef]

11. Zhao, W.; Zhang, X.; Jiang, J.; Wei, N. Spectrum Analysis of Tip-Plane Corona Discharges. Spectrosc. Spectr. Anal. 2003, 23, 955-957.

12. Tang, J.; Liu, Y.; Qiu, Y.; Yuan, J. Relationship Between First Integral Value of Signals of the Optical Method and Charge Quantity of Partial Discharge from Needle-plate Electrode. High Volt. Eng. 2012, 38, 1-8.

13. Xu, Y.; Yu, M.; Cao, X.; Zhen, W.; Hu, S. Optical Pulse Method for Partial Discharge Measurement and the Comparison with Electrical Current Method. High Volt. Eng. 2001, 27, 3-5.

14. Muhr, M.; Schwarz, R. Experience with optical partial discharge detection. Mater. Sci. Pol. 2009, 27, 1139-1146.

15. Li, J.; Han, X.; Liu, Z.; Yao, X. A Novel GIS Partial Discharge Detection Sensor with Integrated Optical and UHF Methods. IEEE Trans. Power Deliv. 2016, 33, 2047-2049. [CrossRef]

16. Wei, N.; Zhang, X.; Cao, H.; He, L. Study of fluorescent fiber sensors for partial discharge detection. J. Tsinghua Univ. Sci. Technol. 2002, 42, 329-332.

17. Kaufhold, M.; Bamji, S.S.; Bulinski, A.T. Optical detection of partial discharges in gas-insulated systems. In Proceedings of the Conference on Electrical Insulation and Dielectric Phenomena (CEIDP), Millbrae, CA, USA, 23 October 1996; Volume 2, pp. 618-623.

18. Maria, L.D.; Bartalesi, D. A Fiber-Optic Multisensor System for Predischarges Detection on Electrical Equipment. IEEE Sens. J. 2012, 12, 207-212. [CrossRef]

19. Huang, J.; Liu, C.; Liu, H.; Ren, M.; Zhang, C.; Dong, M. Characteristics of Partial Discharges at Metal Protrusion Under Oscillating Impulse Voltages in SF6 Gas. High Volt. Eng. 2015, 41, 3872-3880.

20. Ren, M.; Dong, M.; Liu, J. Statistical analysis of partial discharges in SF6 gas via optical detection in various spectral ranges. Energies 2016, 9, 152. [CrossRef]

21. Hikita, M. Fundamental principles and application of diagnosis for GIS using partial discharge measurements. In Proceedings of the IEEE International Conference on Electrical Engineering and Informatics, Bandung, Indonesia, 17-19 July 2011; pp. 1-6.

22. Han, X.; Liu, Z.; Zhang, L.; Li, J.; Yang, J. Simulation for Propagation Characteristics of Optical Signals Excited by Partial Discharge in GIS. J. Xi'an Jiaotong Univ. 2018, 52, 1-7. 
23. Jia, Z.; Gan, D.; Li, J. Design of Insulation Dimension for $\pm 500 \mathrm{kV}$ DC Gas Insulated Transmission Line. Power Syst. Technol. 2011, 35, 192-196.

24. Lei, M.; Chen, L. Discusses on Basic Structure Design of GIL and GIS Busbar. High Volt. Appar. 2013, 49, 128-133.

25. Qian, Y.; Zhang, Y.; Liu, W.; Xu, Y.; Sheng, G.; Jiang, X. Simulation of Partial Discharge Optical Signal Propagation Characteristics in T-type GIS Model. High Volt. Technol. 2019, 45, 1510-1517. 